\title{
Quantum Nature of Dielectric Laser Accelerators
}

\author{
Yuval Adiv $\odot,{ }^{1,2}$ Kangpeng Wang, ${ }^{1,2}$ Raphael Dahan, ${ }^{1,2}$ Payton Broaddus, ${ }^{3}$ Yu Miao $\odot,{ }^{3}$ Dylan Black, ${ }^{3}$ Kenneth Leedle, ${ }^{3}$ \\ Robert L. Byer $\odot,{ }^{4}$ Olav Solgaard $\odot,{ }^{3}$ R. Joel England $\odot,{ }^{5}$ and Ido Kaminer $\oplus^{1,2, *}$ \\ ${ }^{1}$ Department of Electrical and Computer Engineering, \\ Technion-Israel Institute of Technology, Haifa 3200, Israel \\ ${ }^{2}$ Solid State Institute, Technion-Israel Institute of Technology, Haifa 32000, Israel \\ ${ }^{3}$ Department of Electrical Engineering, Stanford University, Stanford, California 94305, USA \\ ${ }^{4}$ Department of Applied Physics, Stanford University, Stanford, California 94305, USA \\ ${ }^{5}$ SLAC National Accelerator Laboratory, 2575 Sand Hill Road, Menlo Park, California 94025, USA
}

(Received 30 May 2021; revised 20 September 2021; accepted 22 September 2021; published 1 December 2021)

\begin{abstract}
Dielectric laser accelerators (DLAs) hold great promise for producing economic and compact on-chip radiation sources. On-chip DLAs benefit from fabrication capabilities of the silicon industry and from breakthroughs in silicon-photonic nanostructures to enhance the interaction between particles and laser fields. Seemingly unrelated recent advances in the quantum interactions of electrons and light have raised interest in the underlying classical-quantum correspondence principle at the foundations of electron acceleration. Here, we present the observation of the underlying quantum nature of DLAs: observing quantized peaks in the electron-energy spectra. Our findings demonstrate quasi-phase-matching between an electron wave function and a light wave, which also demonstrates the role of the quantum wave function in the inverse Smith-Purcell effect. We harness the capabilities of an ultrafast transmission electron microscope (UTEM) to maintain a long electron-light interaction length extending over hundreds of periods of the laser pulse, mediated by a silicon-photonic nanograting DLA. The UTEM is shown as a new platform for characterization of future DLA concepts. The results raise fundamental questions regarding the role of quantum mechanics in DLA design, and more generally about the prospects of manipulating particles' quantum wave functions in accelerator physics.
\end{abstract}

DOI: $10.1103 /$ PhysRevX.11.041042

Subject Areas: Optics, Photonics, Quantum Physics

\section{INTRODUCTION}

Particle accelerators play a key role in many scientific and industrial applications. Accelerators are ubiquitous in modern physics, from the largest accelerators used in highenergy-physics experiments, to commercial accelerators used to implant ions in semiconductors, or in cancer treatments and medical imaging [1,2]. Current high-energy accelerators are based on radio-frequency (rf) technology, but require high investment in space and operational costs. Shrinking the accelerators would open new possibilities for implementing economic and compact radiation sources in numerous applications, e.g., electron diffraction and microscopy, portable medical sources, coherent probes for quantum-information science, etc.

\footnotetext{
*Corresponding author. kaminer@technion.ac.il

Published by the American Physical Society under the terms of the Creative Commons Attribution 4.0 International license. Further distribution of this work must maintain attribution to the author(s) and the published article's title, journal citation, and DOI.
}

A leading approach toward compact-particle accelerators is to facilitate efficient interaction between the particles and light by using dielectric structures and short laser pulses [3-5]. These dielectric laser accelerators (DLAs) operate based on the inverse Smith-Purcell phenomena [6,7], the process of stimulated absorption and emission of photons by an electron that passes close to a periodic structure. Advances in DLA designs could enable optimization of the fundamental electron-laser interaction based on siliconphotonics methods $[4,8]$, as was demonstrated in the recent literature [9-14]. The first successful DLA demonstrations were presented in 2013 showing the acceleration of subrelativistic and relativistic electron beams with energy gradients of a few dozen [6,15] to a few hundred [16] $\mathrm{MeV} / \mathrm{m}$, respectively. Since then, ongoing improvements of DLA designs have been demonstrated, aiming to improve the laser coupling efficiency and increasing the acceleration gradients closer to the $\mathrm{GeV} / \mathrm{m}$ scale, especially with fusedsilica DLAs that have a higher damage threshold [17-24].

Despite the substantial research efforts that have been put into DLAs, all the experiments in the field until now have treated the electron-laser interaction with classical electrodynamics. Hence, the electron was always considered as a 
point particle. Several theory papers described the inverse Smith-Purcell effect with a quantum-mechanical formalism and implied corrections to the DLA process [7,25-31]. However, DLA experiments to date did not access the quantum nature of the process, which requires sufficient electron-beam coherence and energy detection resolution to resolve the individual exchange of photons with the electrons.

Here, we report the first DLA experiment that reveals quantum features in the DLA process, which arise from the underlying electron wave nature [32]. We also provide the quantum theoretical treatment that captures our measurements. Using electron-energy-loss spectroscopy (EELS), we show that the postinteraction electron spectrum, and thus also the inverse Smith-Purcell effect, are inherently quantized by the fundamental photon energy of the incident laser. We also show that such a quantized electron spectrum is accurately described by a generalization of the theory of photon-induced near-field electron microscopy (PINEM) [33-35], which accounts for the electron wave function.

To probe the quantum features of the acceleration process, we utilize the capabilities of an ultrafast transmission electron microscope (UTEM) [33,36-44] and use it to study DLA performance. The UTEM used in this experiment is equipped with an electron-energy spectrometer that can detect energy variations less than the single-photon energy, which enables probing of the quantum features of the DLA process. This demonstration showcases the UTEM as another effective setup to characterize new DLA designs, for example, by measuring the DLA response to changes in the excited laser wavelength and initial electron-beam energy. Furthermore, our results raise intriguing questions regarding the necessity of a quantum theory for future design and improvement of certain DLA performances.

\section{THE EXPERIMENTAL SETUP}

The UTEM consists of a TEM that is triggered by a femtosecond laser pulse simultaneously with a second pulse illuminating the investigated sample. This system is capable of conducting pump-probe experiments to study phenomena with nanometer spatial resolution and femtosecond temporal resolution simultaneously [36]. Figure 1(a) depicts the schematics of the UTEM we use for the DLA study. The electron probe pulse is photoemitted by a femtosecond UV pulse that hits a $\mathrm{LaB}_{6}$ tip. A set of tunable electromagnetic lenses control the electronpulse divergence and spot size at the interaction point. Different from most UTEM experiments [33,37-43], we use the electromagnetic lenses to create a highly collimated (a)

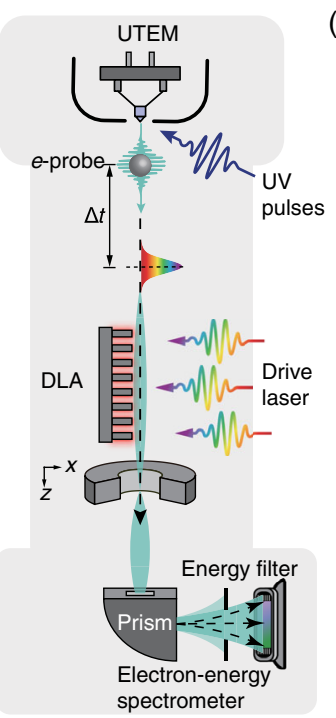

(b) $\Gamma^{y}$

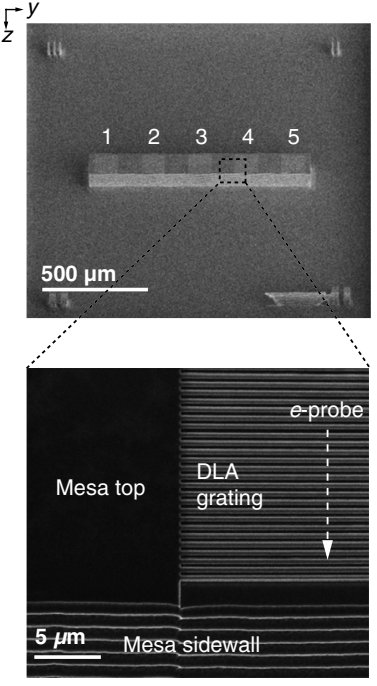

(c)

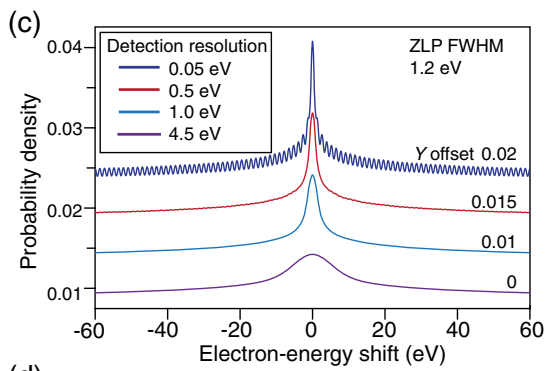

(d)

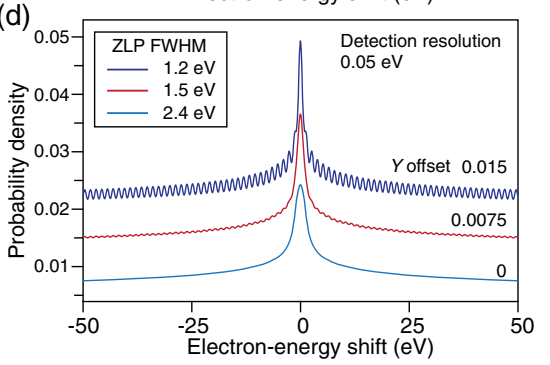

FIG. 1. Study of DLA in the UTEM. (a) A schematic of the UTEM and its special mode of operation providing the platform to study electron acceleration. (b) Scanning-electron-microscopy image of the DLA structure in our experiment. This DLA is a one-sided siliconbased grating with a period of $655 \mathrm{~nm}$, teeth height of $195 \mathrm{~nm}, 45 \%$ duty cycle, and a total length of $89 \mu \mathrm{m}$ designed to mediate the interaction between a 950-nm laser pulse and a 195-keV electron pulse. The top panel depicts a set of five similar gratings separated by a flat mesa square formed for alignment convenience; below is an enlargement of the border between such a grating and a flat mesa area. (c) Quantum theory of the electron-energy spectrum after DLA interaction, calculated with four different detection resolutions. The resolution of $4.5 \mathrm{eV}$ is the best that was used in DLA experiments till now [17], to the best of our knowledge, and it is not sufficient for probing the quantum nature of the interaction. The three other resolutions of $1,0.5$, and $0.05 \mathrm{eV}$ are available in state-of-the-art electron spectrometers in electron microscopes and used in the UTEM setup in this work. Yet, only the finer resolution among them is sufficient to unveil quantum features in the spectrum (for the given parameters), as we also see experimentally below. (d) Theoretical electronenergy spectrum after DLA interaction calculated with three different initial energy spreads of the electron beam. The quantum nature of the interaction is revealed only for an electron beam that is highly coherent (approximately monoenergetic). 
electron beam that grazes the sample [44] [Fig. 1(a)] rather than penetrate it. The highly collimated nature of the electron beam allows close approach to the sample, leading to stronger electron-light coupling and to a higher gradient. The second femtosecond laser pulse, the pump, is incident on the sample surface at a controllable angle. The DLA sample is held by a TEM holder whose position relative to the electron beam and tilt angle are determined with nanometer resolution and a step of $0.1^{\circ}$, respectively. After the interaction, the electron probe reaches the energy spectrometer for analysis of the energy modulation due to the interaction.

The DLA sample in our experiment is a one-sided silicon grating [Fig. 1(b)] which is optimized for the inverse SmithPurcell interaction with a $195-\mathrm{keV}$ electron probe $(\beta=0.69)$ and a pump excitation at $950 \mathrm{~nm}$ (from an optical parametric amplifier) incident perpendicular to the surface. The decay length (1/e of its maximal value) of the excited near-field response along the coordinate perpendicular to the plane of the DLA device is $144 \mathrm{~nm}$. In this part of the experiment, the measured laser spot size is $50 \mu \mathrm{m}$ (1/e of the electric field), and the measured electron beam convergence angle is $0.66 \mathrm{mrad}$. The electron-beam and laser-pulse durations and the electron-beam spot size are changed during the experiment, and the values are mentioned next to the corresponding measurements below.

The main advantage of the UTEM for DLA studies, in comparison with other experimental approaches, is the highresolution spectrometer. This spectrometer can reach an energy resolution of $0.01 \mathrm{eV}$, enabling the detection of events associated with the exchange of single photons with an electron [Fig. 1(c)]. As a result, the practical limitation for probing quantum features through EELS measurements is the initial energy spread of the electron [also called the zeroloss peak (ZLP) width; Fig. 1(d)]. The electron pulse in the UTEM can achieve an energy spread of approximately $0.5 \mathrm{eV}$ at full width at half maximum (FWHM) comparable to the best performance that could be achieved in some other DLA experiments (which were limited by the spectrometer energy resolution rather than the electron-energy spread) $[17,18,23]$. This level of coherence meets the conditions for investigating quantum features as shown below.

\section{EXPERIMENTAL RESULTS AND ANALYSIS}

The laser pulse that excites the DLA creates the near field with which the electron pulse interacts [simulation in Fig. 2(a)]. We can describe the coupling of this interaction by a unitless parameter $g$ simulated in Fig. 2(b). This coupling strength is determined by the near-field distribution, and we discuss it in detail below. Figure 2(c) depicts the results of the electron-energy postinteraction spectrum as a function of the incident laser wavelength, showing that the maximal response occurs at $950 \mathrm{~nm}$ as designed. This result conforms with the prediction of the inverse Smith-Purcell effect of a critical wavelength dependence for optimal interaction and hence fulfills the synchronicity condition [45] given by the inverse Smith-Purcell theory $\lambda_{0}=(\Lambda / n)[(1 / \beta)-\cos \theta]$, where $\lambda_{0}$ is the wavelength of the excitation laser, $\Lambda$ is the grating period, $n$ the harmonic order, $\beta$ the relative electron velocity, and $\theta$ the angle relative to the electron velocity. The laser is normally incident in our case $(\theta=0)$, and thus the synchronicity condition can be written as $\Lambda=n \beta \lambda_{0}$. Thus, the wavelength scan validates the prediction of the inverse Smith-Purcell theory and demonstrates the accuracy of the fabricated grating periodicity.

Figure 3 shows the energy spectrum of each electron after interaction with the DLA near field. The energy spectrum is acquired by repeating the experiment to accumulate a histogram of single-electron detection events. In this spectrum, the measured laser-pulse duration $(1 / e$ of the electric field) is $102 \mathrm{fs}$, the root-mean-square values of the electron bunch duration (fitted) and electron spot size at the interaction point (measured) are $159 \mathrm{fs}$ and $159 \mathrm{~nm}$, respectively (see Supplemental Material [46] for detailed information of the experimental parameters). The recorded initial electron-energy spread is $3.5 \mathrm{eV}$ at FWHM (left inset), and the spectrum bandwidth after the interaction is $4000 \mathrm{eV}$, equally stretched to the loss and gain sides, representing deceleration and acceleration of the electrons, respectively. We measure the maximum acceleration gradient $G=\Delta E_{\max } / L_{\max }=67 \mathrm{MeV} / m$, where $\Delta E_{\max }=$ $2 \mathrm{keV}$ is the maximum energy change, and $L_{\max }=$ $30 \mu \mathrm{m}$ is the interaction length as determined by the electron velocity and by the laser spot size and duration [see Eq. (3) in Ref. [17] ].

We note that higher acceleration gradients have already been achieved [16-23]; however, we use a relatively low-peak-field amplitude $(0.7 \mathrm{GV} / \mathrm{m}$ compared with $1-3.5 \mathrm{GV} / \mathrm{m}$ in Refs. [6,16-19,21-23]), and we do not yet optimize the DLA structure. Moreover, the DLA used in this experiment does not include special features (e.g., couplers, integrated waveguides [24], or distributed Bragg mirrors [23]) to enhance the efficiency of the energy transfer from the laser pulse to the near-field mode that interacts with the electron. Improving the DLA design and specifically improving coupling into the DLA mode are expected to yield a higher acceleration gradient and more efficient energy transfer from the laser to the electron. Improving this energy transfer is also highly beneficial for quantum experiments, as it increases the probability of interaction between a single electron and a single photon, allowing for more efficient operation at lower electron currents and/or lower field power. The acceleration spectrum depicted in Fig. 3 is well described by both the classical DLA theory (pointlike electron) and by a quantum treatment that accounts for the electron wave function (this theoretical treatment is discussed thoroughly below in the text).

Figure 4 shows a different postinteraction electron spectrum that reveals the quantum nature of the acceleration process. For this measurement, we stretch the laser-pulse duration to $382 \mathrm{fs}$ (1/e of the electric field). 
(a)

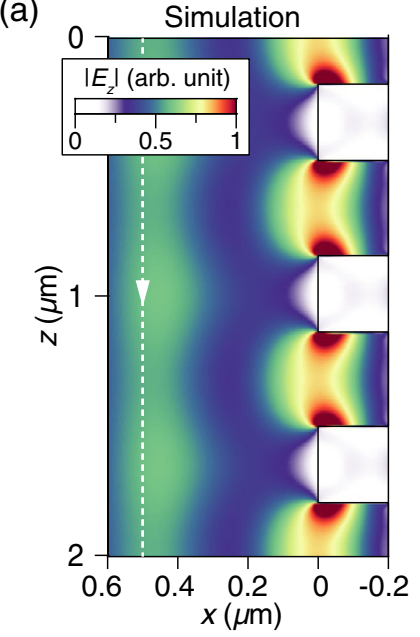

(b)

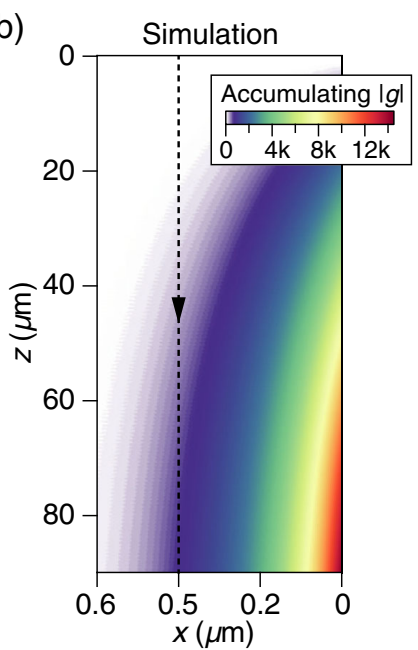

(c)

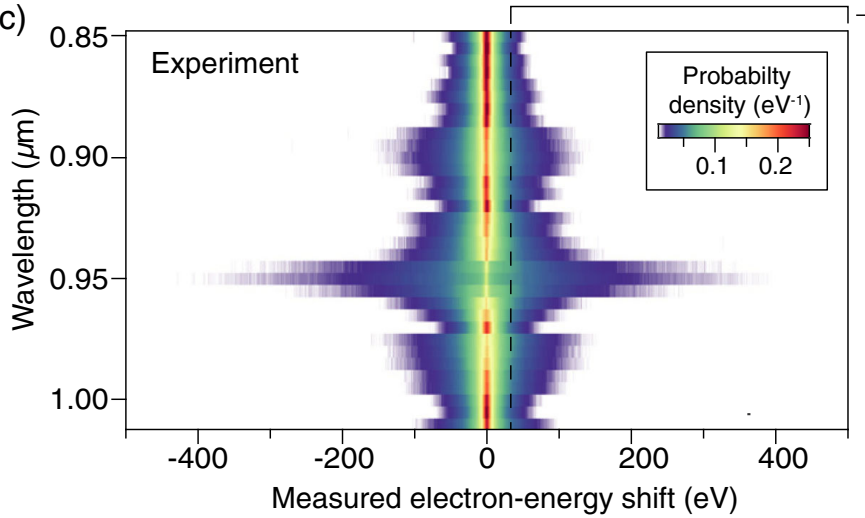

Simulation

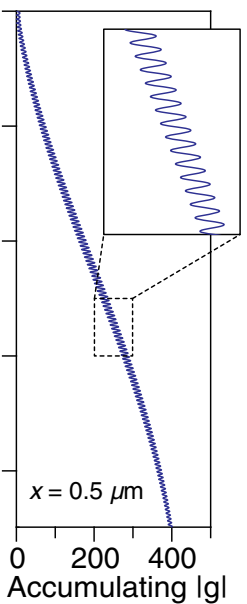

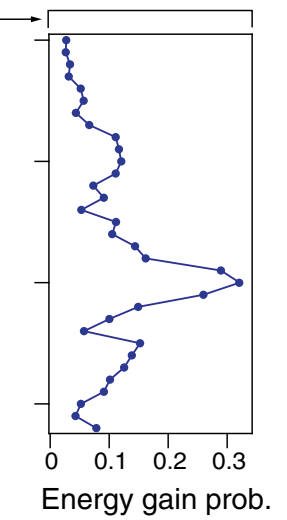

FIG. 2. The DLA near-field simulation and its measured wavelength dependence. (a) A finite-difference time-domain simulation of the electric field (polarization parallel to the electron trajectory in an absolute value), which causes the electron acceleration. The DLA structure alters the near-field pattern to achieve both a longitudinal field component and a phase-matching condition that facilitate efficient interaction. Note that the range $0<\mathrm{x}<0.2 \mu \mathrm{m}$ is inside the grating and therefore cannot be accessed by the electrons. Most of the presented range $0<\mathrm{x}<0.6 \mu \mathrm{m}$ is outside the grating and accessible by the electrons. (b) The left panel shows the integrated coupling strength accumulated along the interaction of the electron with the DLA as simulated using Eq. (2). The right panel shows a cross section of this coupling strength at $\mathrm{x}=0.5 \mu \mathrm{m}$, which is $500 \mathrm{~nm}$ away from the grating surface, approximately the distance of the center of the electron beam at the measurement depicted in Fig. 3 (see the Supplemental Material [46] for these measurement parameters). The inset highlights the oscillations of the coupling strength resulting from the grating near-field periodicity. (c) The left panel depicts a wavelength scan response of the DLA structure-EELS measurements for different wavelengths between 850 and $1010 \mathrm{~nm}$ with a 5-nm step — showing maximal interaction in the wavelength of $950 \mathrm{~nm}$, in agreement with the theoretical design. This measurement tests the structure wavelength response and shows the accuracy of the fabricated periodicity. For the right panel, we sum the number of electrons that gain at least $6 \mathrm{eV}$ (i.e., outside of the ZLP) for each wavelength, corresponding to the probability of electrons to gain energy from the DLA near field, showing a peak at the resonant wavelength.

The root-mean-square values (fitted) of the electron bunch duration and electron spot size at the interaction point are $200 \mathrm{fs}$ and $445 \mathrm{~nm}$, respectively (see Supplemental Material [46] for detailed information of the experimental parameters). The measured spectrum (blue dots) is comprised of individual peaks placed at multiples of the fundamental photon energy of the incident laser $\hbar \omega_{0}\left(\omega_{0}\right.$ is the central laser-pulse frequency). Each peak corresponds to stimulated emission (if appearing on the loss side) or absorption (if appearing on the gain side) of individual photons by each individual electron through the inverse Smith-Purcell effect, i.e., deceleration and acceleration, respectively. To probe these quantized features, we increase the dispersion of our spectrometer to $0.05 \mathrm{eV}$ per channel and tune the electron-beam parameters to a measured electron initial energy spread (ZLP width) of $1.15 \mathrm{eV}$ at FWHM (equivalent $0.5-\mathrm{eV}$ rms value; see gray transparent background in the top panel of Fig. 4). This initial energy spread is lower than the energy of a 950-nm photon, which is approximately $1.3 \mathrm{eV}$. Thus, when combined with the fine detection resolution, we observe the quantum features of the acceleration process. We expect all DLA experiments to have such features, which have so far remained below the detection capabilities.

This recorded spectrum implies that the DLA process, and hence, the inverse Smith-Purcell phenomena, depends 


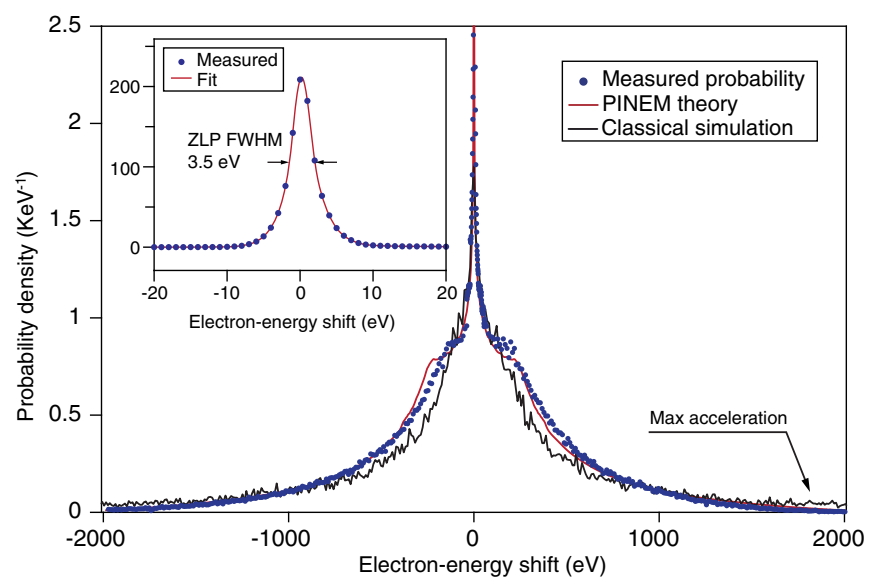

FIG. 3. Measurement of DLA in the UTEM and comparison with theory. The EELS measurement shows the electron acceleration and deceleration. The recorded initial energy spread of the electron pulse is $3.5 \mathrm{eV}$ at FWHM (left inset), while the measured postinteraction energy spectrum has a bandwidth of $4000 \mathrm{eV}$ (blue dots). The maximum achieved acceleration is $2000 \mathrm{eV}$ determined by the point at which the signal approximately reaches the noise level of the spectrometer. For this measurement, we set our spectrometer dispersion at $1 \mathrm{eV}$ per channel (total channel number: 2000), record each side of the spectrum (gain and loss) separately with a small overlap, and then splice them together. This coarse dispersion allows us to capture the full spectrum with two shots only, but on the other hand, it measures a broader ZLP than it actually is. As a result, the EELS dispersion conceals the quantum features (see Fig. 4). We show that both the particle-tracking simulation, which is based on the classical DLA theory (solid black line), and the PINEM theory (solid red line) produce good predictions of the measured spectrum. The horizontal error for each point on the measured curves is determined by the measured initial energy width of the electron beam $(1.49 \mathrm{eV})$ and by the detector resolution $( \pm 1 \mathrm{eV})$.

on the quantum wave nature of the electron. A classical description, as was used in all DLA experiments so far, is not sufficient to explain this result, and a more advanced quantum theory is needed.

The quantum-mechanical theory of electron wave functions interacting with (classical) laser fields is described by the approach of PINEM $[34,35]$. This theory was originally developed to describe interactions that are short in time and space, whereas in our case, the electron maintains its interaction with the DLA nanostructure along tens of microns and for hundreds of femtoseconds. An extended PINEM theory has been developed to capture the full spatiotemporal relation between the electron and the laser pulse for a prolonged interaction duration and an extended interaction length [44]. Using this theory, the electronenergy spectrum after the interaction is given by

$$
\begin{aligned}
\rho(u, x, y, t)= & \rho_{0}(u, x, y, t) \\
& * \sum_{\ell=-\infty}^{\infty} J_{\ell}^{2}(2|g(x, y, t)|) \delta\left(u-\ell \hbar \omega_{0}\right),
\end{aligned}
$$
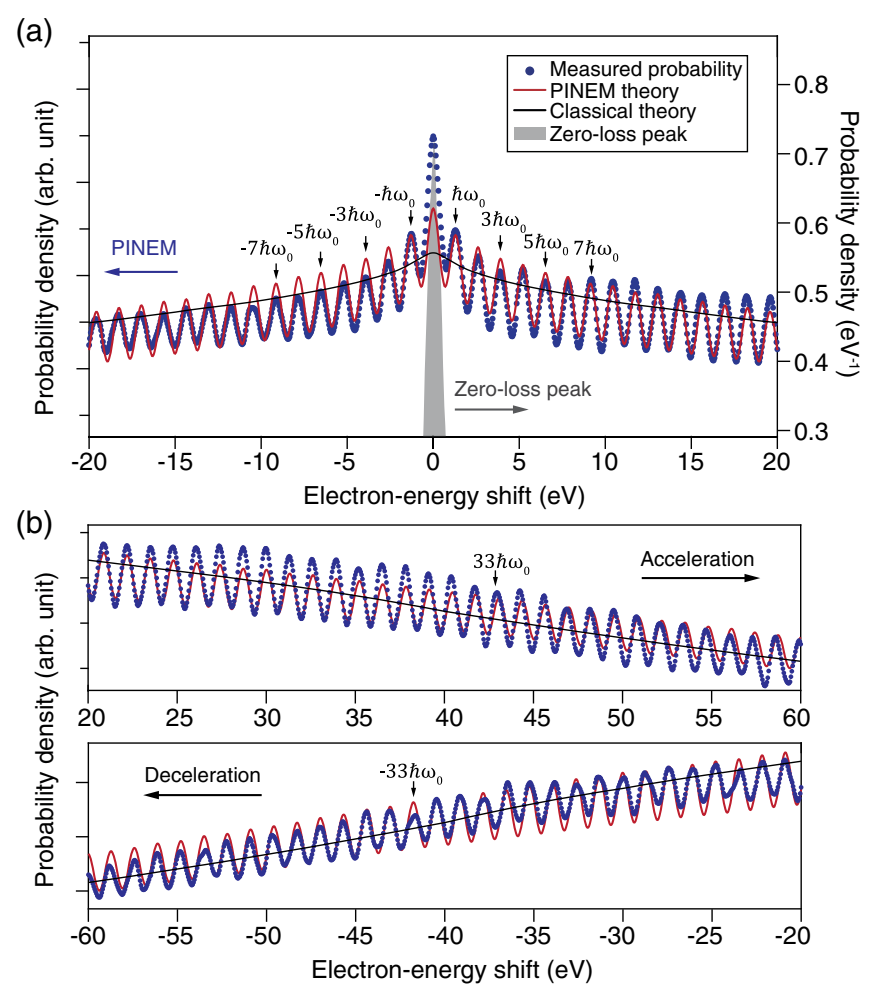

FIG. 4 The quantum nature of electron-laser acceleration observed in a DLA. The electron-energy spectrum after the interaction with the DLA near field shows its quantized nature, as it oscillates with the periodicity of the fundamental photon energy of the incident laser. To reveal this quantum behavior, we perform the same EELS measurement as described in Fig. 3, but we use a finer detection resolution of $0.05 \mathrm{eV}$ which leads to a measured electron coherency of $1.15 \mathrm{eV}$ at FWHM (rms of $0.5 \mathrm{eV}$ ). The important change in the detection resolution enables us to probe features that are below single-photon energy and to reveal the fine features in the spectral shape of each accelerated electron. In the above, we also stretch the duration of the laser pulse relative to the case in Fig. 3 to cover more of the electron wave function by the laser pulse. This causes a larger portion of the electrons to interact, resulting in a lower ZLP (see Supplemental Material [46] for parameters comparison and the relations between the different timescales in the experiment). The theoretical spectrum produced by the extended PINEM theory [solid red line, Eqs. (1) and (2)] matches well with the measured data, unlike the classical theory (black solid line), which is calculated with the classical formula appearing in the text below Eq. (1) (see also the Supplemental Material [46]). The horizontal error for each point on the measured curves is determined by the measured initial energy width of the electron beam $(0.5 \mathrm{eV})$ and by the detector resolution $( \pm 0.05 \mathrm{eV})$.

where $u$ denotes the electron-energy change, and $\rho_{0}$ is the incoherent contributions to the electron, i.e., its initial energy distribution, transverse spatial distribution, and temporal envelope. This incoherent part is convoluted $(*)$ with the coherent part contribution over both energy and time. The coherent contribution of the electron 
spectrum contains $J_{\ell}$ for a Bessel function of order $l$, and $\delta(u)$ for the Dirac delta function. The classical analog of Eq. (1) reads $\rho=\rho_{0} *\left[\pi \sqrt{\left(2|g| \hbar \omega_{0}\right)^{2}-u^{2}}\right]^{-1}$ (see Supplemental Material [46] for the derivation).

The time-dependent unitless coupling strength denoted in Eq. (1) as $g$ is given by

$$
g(x, y, t)=\frac{q_{\mathrm{e}}}{\hbar \omega_{0}} \int_{-\infty}^{\infty} \tilde{E}_{\mathrm{z}}\left(\boldsymbol{r}, t+\frac{z}{v_{\mathrm{e}}}\right) e^{-i\left(\omega_{0} / v_{\mathrm{e}}\right) z} d z,
$$

where $q_{\mathrm{e}}$ is the elementary charge, $\tilde{E}_{\mathrm{z}}$ is the electric field phasor component along the electron trajectory, and $v_{\mathrm{e}}$ is the electron velocity. This unitless coupling strength $g$, when multiplied by the photon energy and divided by the interaction length is equivalent to the acceleration gradient $G$ of the DLA experiment [1].

Seeking the correspondence between the PINEM theory and the recorded quantized spectrum, we fit the electronand laser-pulse parameters using a programming solver (see Supplemental Material [46]). We summarize the resulting parameters from the fit and compare them to direct measurements, if available, of the same parameters in Tables I and II in the Supplemental Material [46]. The fitted PINEM spectrum (solid red line in Fig. 4) describes the measured data with good correspondence, and all the fit parameters are within the error boundaries of the direct measurements and known characteristics of our setup (see Supplemental Material [46] for further details). The successful correspondence of the PINEM theory comes from the electron description as a wave function, as reflected by the presence of the Bessel functions in Eq. (1). The oscillatory nature of these Bessel functions, which is totally absent from the classical prediction, enables the description of the quantized spectrum. We note that the measured spectrum exhibits a certain asymmetry between the gain and loss sides. This effect is not captured by the conventional PINEM theory and originates from the free-space propagation of the modulated electron wave function. A detailed explanation of this phenomena is a subject for future work.

\section{DISCUSSION AND OUTLOOK}

Our experiment shows that the DLA process contains quantum features that are influenced by the electron wave function. This result is also the demonstration of the quantum nature of the inverse Smith-Purcell effect, as manifested in the EELS. The recorded quantized spectrum implies that the single-electron wave function splits into several thousand distinct peaks of different energy, all part of the same wave function-coherent with one another. Such a multipeaked wave function forms a broadband energy comb. Thus, a single electron occupies thousands of energy states simultaneously, constituting in the time domain a temporal comb of subattosecond pulses. The classical theory used until now in DLA designs and experiments is not sufficient to explain the quantized spectrum, and we show how the extended PINEM theory is suitable for this purpose.

Importantly, the results of this study show that electron interaction with DLAs can maintain quantum coherence for timescales of a hundred laser cycles. This happens despite the involvement of different interaction mechanisms (e.g., bulk plasmons, phonons, bremsstrahlung, etc.) that can cause loss of coherency. Previous PINEM experiments that showed quantum coherence used thin structures [33,37-43] (tens to hundreds of nanometers) in which the typical decoherence timescales are longer than the interaction duration. In addition, we show that the interaction maintains its quantum coherence not only for a long duration but also for strong interactions. There is an open question about the coherence of strong interactions, where the physics of quantum walk that describes such interactions [38] has to be modified by a gradual broadening of the electron-energy levels due to the coherent uncertainty of the driving laser [47]. While our work does not resolve this question in its entirety, it shows that coherence remains even for exchange of hundreds of photons. This implication may be also useful beyond DLAs, for other silicon-photonic platforms.

Looking forward, it is important to identify the conditions at which the underlying quantum behavior of the interaction will necessitate reconsidering current DLA designs. The quantum description of the electron provides additional degrees of freedom to the acceleration process, which could potentially provide new opportunities for better accelerators. Importantly, for many DLA applications, higher electron densities are needed, and it would thus become necessary to study how the quantum nature of DLA extends to multielectron scenarios. A full treatment would require a challenging many-body quantum simulation, and it is not yet known whether the quantum features (e.g., discrete energy peaks) will remain. Certain meanfield approximations were predicted in the past to preserve quantum wave-function effects in the case of a many-body electron beam [48]. For instance, we expect a significant influence on performance due to quantum behavior when prebunching is used as part of the acceleration scheme [49]. In the quantum case, the bunching can happen on a single electron that becomes a comb of attosecond pulses after a DLA interaction. The resulted attosecond electron pulse can then be used for producing net acceleration [50] or for time-resolved atomic-scale dynamics [51-53]. Importantly, the quantum description predicts bunching dynamics that are different from that of a classical theory, and the quantum-mechanical uncertainty principle poses a lower bound on the achievable bunch duration [54]. We envision the DLA as a platform for efficient preparation of attosecond electron combs for spectroscopy purposes, as complementary to the original goal of acceleration. 
Besides the integral advantage of the high-resolution spectrometer of the UTEM in studying the quantum nature of the DLA interaction, we also demonstrate in this work that the UTEM is a valuable platform to characterize different properties of DLAs. Follow-up experiments may include improved laser coupling, waveguided-coupled DLAs $[24,55,56]$, or new acceleration schemes such as metasurface laser accelerators [57,58].

Finally, the coupling strength of a DLA interaction has shown how quasi-phase-matching of an electron wave function and a light wave can result in a fairly efficient interaction. This efficient interaction suggests that optimized DLAs could bring us to the desired regime of singleelectron-single-photon interaction [59]. This way, DLAs may open the way toward free-electron quantum-optics experiments, which require the quantization of the electromagnetic field [60,61]. As a future goal, efficient DLA interactions may enable access to regimes where nonclassical light can shape an electron wave function [62] and even allow measurements and reconstruction of the quantum state of light using free electrons [63,64].

\section{ACKNOWLEDGMENTS}

The experiments were performed on the UTEM of the AdQuanta group of I. K., which is installed inside the electron microscopy center (MIKA) of the Department of Materials Science and Engineering at the Technion. We thank IDES and especially S. T. Park for support and advice. We also thank Yahel Barak for creating the accompany image for this manuscript. I. K. and his students gratefully acknowledge the generous support of R. Magid and R. Magid, who have made it possible to carry out this experiment at the UTEM lab at the Technion. This work was supported by the European Union's Horizon 2020 research and innovation program under Grant agreement No. 851780-ERC-NanoEP, the Israel Science Foundation (Grant No. 830/19), the Binational USA-Israel Science Foundation (BSF) 2018288. Parts of this work is supported by Gordon and Betty Moore Foundation (GBMF4744).

[1] L. Schachter, Beam-Wave Interaction in Periodic and Quasi-Periodic Structures (American Institute of Physics, New York, 1997), https://www.springer.com/gp/book/ 9783662033982.

[2] R. W. Hamm and M. E. Hamm, Industrial Accelerators and Their Applications (World Scientific, Singapore, 2012), https://www.worldscientific.com/worldscibooks/10.1142/ 7745.

[3] R. J. England, R. J. Noble, K. Bane, D. H. Dowell, C. K. Ng, J. E. Spencer, S. Tantawi, Z. Wu, R. L. Byer, E. Peralta, K. Soong, C. M. Chang, B. Montazeri, S. J. Wolf, B. Cowan, J. Dawson, W. Gai, P. Hommelhoff, Y. C. Huang, C. Jing et al., Dielectric Laser Accelerators, Rev. Mod. Phys. 86, 1337 (2014).
[4] J. Breuer, J. McNeur, and P. Hommelhoff, Dielectric Laser Acceleration of Electrons in the Vicinity of Single and Double Grating Structures-Theory and Simulations, J. Phys. B 47, 234004 (2014).

[5] K. P. Wootton, J. McNeur, and K. J. Leedle, Dielectric Laser Accelerators: Designs, Experiments, and Applications, Rev. Accel. Sci. Techol. 09, 105 (2016).

[6] J. Breuer and P. Hommelhoff, Laser-Based Acceleration of Nonrelativistic Electrons at a Dielectric Structure, Phys. Rev. Lett. 111, 134803 (2013).

[7] N. Talebi, Schrödinger Electrons Interacting with Optical Gratings: Quantum Mechanical Study of the Inverse Smith-Purcell Effect, New J. Phys. 18, 123006 (2016).

[8] A. Aimidula, M. A. Bake, F. Wan, B. S. Xie, C. P. Welsch, G. Xia, O. Mete, M. Uesaka, Y. Matsumura, M. Yoshida, and K. Koyama, Numerically Optimized Structures for Dielectric Asymmetric Dual-Grating Laser Accelerators, Phys. Plasmas 21, 023110 (2014).

[9] X. E. Lin, Photonic Band Gap Fiber Accelerator, Phys. Rev. ST Accel. Beams 4, 051301 (2001).

[10] B. M. Cowan, Two-Dimensional Photonic Crystal Accelerator Structures, Phys. Rev. ST Accel. Beams 6, 101301 (2003).

[11] A. Mizrahi and L. Schächter, Optical Bragg Accelerators, Phys. Rev. E 70, 016505 (2004).

[12] Z. Zhang, S. G. Tantawi, and R. D. Ruth, Distributed Grating-Assisted Coupler for Optical All-Dielectric Electron Accelerator, Phys. Rev. ST Accel. Beams 8, 071302 (2005).

[13] T. Plettner, P. P. Lu, and R. L. Byer, Proposed Few-Optical Cycle Laser-Driven Particle Accelerator Structure, Phys. Rev. ST Accel. Beams 9, 111301 (2006).

[14] B. M. Cowan, Three-Dimensional Dielectric Photonic Crystal Structures for Laser-Driven Acceleration, Phys. Rev. ST Accel. Beams 11, 011301 (2008).

[15] J. Breuer, R. Graf, A. Apolonski, and P. Hommelhoff, Dielectric Laser Acceleration of Nonrelativistic Electrons at a Single Fused Silica Grating Structure: Experimental Part, Phys. Rev. ST Accel. Beams 17, 021301 (2014).

[16] E. A. Peralta, K. Soong, R. J. England, E. R. Colby, Z. Wu, B. Montazeri, C. McGuinness, J. McNeur, K. J. Leedle, D. Walz, E. B. Sozer, B. Cowan, B. Schwartz, G. Travish, and R. L. Byer, Demonstration of Electron Acceleration in a Laser-Driven Dielectric Microstructure, Nature (London) 503, 91 (2013).

[17] K. J. Leedle, R. F. Pease, R. L. Byer, and J. S. Harris, Laser Acceleration and Deflection of $96.3 \mathrm{keV}$ Electrons with a Silicon Dielectric Structure, Optica 2, 158 (2015).

[18] K. J. Leedle, A. Ceballos, H. Deng, O. Solgaard, R. Fabian Pease, R. L. Byer, and J. S. Harris, Dielectric Laser Acceleration of Sub-100 KeV Electrons with Silicon Dual-Pillar Grating Structures, Opt. Lett. 40, 4344 (2015).

[19] K. P. Wootton, Z. Wu, B. M. Cowan, A. Hanuka, I. V. Makasyuk, E. A. Peralta, K. Soong, R. L. Byer, and R. J. England, Demonstration of Acceleration of Relativistic Electrons at a Dielectric Microstructure Using Femtosecond Laser Pulses, Opt. Lett. 41, 2696 (2016).

[20] K. J. Leedle, D. S. Black, Y. Miao, K. E. Urbanek, A. Ceballos, H. Deng, J. S. Harris, O. Solgaard, and R. L. Byer, Phase-Dependent Dielectric Laser Acceleration of 
$99 \mathrm{keV}$ Electrons with Symmetrically Driven Silicon Dual Pillar Gratings, Opt. Lett. 43, 2181 (2018).

[21] D. Cesar, S. Custodio, J. Maxson, P. Musumeci, X. Shen, E. Threlkeld, R. J. England, A. Hanuka, I. V. Makasyuk, E. A. Peralta, K. P. Wootton, and Z. Wu, High-Field Nonlinear Optical Response and Phase Control in a Dielectric Laser Accelerator, Commun. Phys. 1, 46 (2018).

[22] D. Cesar, J. Maxson, P. Musumeci, X. Shen, R. J. England, K. P. Wootton, S. Tan, and D. Cesar, Enhanced Energy Gain in a Dielectric Laser Accelerator Using a Tilted Pulse Front Laser, Opt. Express 26, 29216 (2018).

[23] P. Yousefi, N. Schönenberger, J. Mcneur, M. Kozák, U. Niedermayer, and P. Hommelhoff, Dielectric Laser Electron Acceleration in a Dual Pillar Grating with a Distributed Bragg Reflector, Opt. Lett. 44, 1520 (2019).

[24] N. V. Sapra, K. Y. Yang, D. Vercruysse, K. J. Leedle, D. S. Black, R. J. England, L. Su, R. Trivedi, Y. Miao, O. Solgaard, R. L. Byer, and J. Vuc, On-Chip Integrated Laser-Driven Particle Accelerator, Science 367, 79 (2020).

[25] N. Talebi, Interaction of Electron Beams with Optical Nanostructures and Metamaterials: From Coherent Photon Sources towards Shaping the Wave Function, J. Opt. 19, 103001 (2017).

[26] S. Tsesses, G. Bartal, and I. Kaminer, Light Generation via Quantum Interaction of Electrons with Periodic Nanostructures, Phys. Rev. A 95, 013832 (2017).

[27] A. Gover and Y. Pan, Dimension-Dependent Stimulated Radiative Interaction of a Single Electron Quantum Wavepacket, Phys. Lett. A 382, 1550 (2018).

[28] Y. Pan and A. Gover, Spontaneous and Stimulated Radiative Emission of Modulated Free-Electron Quantum Wavepackets-Semiclassical Analysis, J. Phys. Commun. 2, 115026 (2018).

[29] Y. Pan and A. Gover, Spontaneous and Stimulated Emissions of a Preformed Quantum Free-Electron Wave Function, Phys. Rev. A 99, 052107 (2019).

[30] Y. Pan, B. Zhang, and A. Gover, Anomalous PhotonInduced Near-Field Electron Microscopy, Phys. Rev. Lett. 122, 183204 (2019).

[31] Y. Pan, E. Cohen, E. Karimi, A. Gover, I. Kaminer, and Y. Aharonov, Weak Measurement, Projective Measurement and Quantum-to-Classical Transitions in Electron-Photon Interactions, arXiv:1910.11685v2.

[32] Y. Adiv, K. Wang, R. Dahan, P. Broaddus, Y. Miao, D. Black, K. Leedle, O. Solgaard, J. England, and I. Kaminer, in Proceedings of the Conference on Lasers and Electro-Optics, Optical Society of America (2020), p. SW4G.4, https://www.osapublishing.org/abstract.cfm? uri=CLEO_SI-2020-SW4G.4.

[33] B. Barwick, D. J. Flannigan, and A. H. Zewail, PhotonInduced Near-Field Electron Microscopy, Nature (London) 462, 902 (2009).

[34] F. J. Garcia De Abajo, A. Asenjo-Garcia, and M. Kociak, Multiphoton Absorption and Emission by Interaction of Swift Electrons with Evanescent Light Fields, Nano Lett. 10, 1859 (2010).

[35] S. T. Park, M. Lin, and A. H. Zewail, Photon-Induced NearField Electron Microscopy (PINEM): Theoretical and Experimental, New J. Phys. 12, 123028 (2010).
[36] A.H. Zewail, Four-Dimensional Electron Microscopy, Science 328, 187 (2010).

[37] L. Piazza, T. T. A. Lummen, E. Quiñonez, Y. Murooka, B. W. Reed, B. Barwick, and F. Carbone, Simultaneous Observation of the Quantization and the Interference Pattern of a Plasmonic Near-Field, Nat. Commun. 6, 6407 (2015).

[38] A. Feist, K. E. Echternkamp, J. Schauss, S. V. Yalunin, S. Schäfer, and C. Ropers, Quantum Coherent Optical Phase Modulation in an Ultrafast Transmission Electron Microscope, Nature (London) 521, 200 (2015).

[39] Y. Morimoto and P. Baum, Diffraction and Microscopy with Attosecond Electron Pulse Trains, Nat. Phys. 14, 252 (2018).

[40] G. M. Vanacore, I. Madan, G. Berruto, K. Wang, E. Pomarico, R. J. Lamb, D. McGrouther, I. Kaminer, B. Barwick, F. J. García De Abajo, and F. Carbone, Attosecond Coherent Control of Free-Electron Wave Functions Using Semi-Infinite Light Fields, Nat. Commun. 9, 2694 (2018).

[41] G. M. Vanacore, G. Berruto, I. Madan, E. Pomarico, P. Biagioni, R. J. Lamb, D. McGrouther, O. Reinhardt, I. Kaminer, B. Barwick, H. Larocque, V. Grillo, E. Karimi, F. J. García de Abajo, and F. Carbone, Ultrafast Generation and Control of an Electron Vortex Beam via Chiral Plasmonic Near Fields, Nat. Mater. 18, 573 (2019).

[42] K. Wang, R. Dahan, M. Shentcis, Y. Kauffmann, A. Ben Hayun, O. Reinhardt, S. Tsesses, and I. Kaminer, Coherent Interaction between Free Electrons and a Photonic Cavity, Nature (London) 582, 50 (2020).

[43] O. Kfir, H. Lourenço-Martins, G. Storeck, M. Sivis, T. R. Harvey, T. J. Kippenberg, A. Feist, and C. Ropers, Controlling Free Electrons with Optical WhisperingGallery Modes, Nature (London) 582, 46 (2020).

[44] R. Dahan, S. Nehemia, M. Shentcis, O. Reinhardt, Y. Adiv, X. Shi, O. Be, M. H. Lynch, Y. Kurman, K. Wang, and I. Kaminer, Resonant Phase-Matching between a Light Wave and a Free-Electron Wavefunction, Nat. Phys. 16, 1123 (2020).

[45] U. Niedermayer, T. Egenolf, and O. Boine-Frankenheim, Beam Dynamics Analysis of Dielectric Laser Acceleration Using a Fast 6D Tracking Scheme, Phys. Rev. Accel. Beams 20, 111302 (2017).

[46] See Supplemental Material at http://link.aps.org/supplemental/ 10.1103/PhysRevX.11.041042 for details about the experimental system and data analysis procedures, as well as for the derivation of the classical equation for the electron spectrum after the interaction.

[47] O. Reinhardt, S. Tsesses, M. Shentcis, K. Wang, S. Nehemia, R. Dahan, and I. Kaminer, in Proceedings of the Conference on Lasers and Electro-Optics, Optical Society of America (2020), p. FTu3D.6, https://www .osapublishing.org/abstract.cfm?uri=CLEO_QELS-2020FTu3D.6.

[48] M. Mutzafi, I. Kaminer, G. Harari, and M. Segev, NonDiffracting Multi-Electron Vortex Beams Balancing Their Electron-Electron Interactions, Nat. Commun. 8, 650 (2017).

[49] N. Schönenberger, A. Mittelbach, P. Yousefi, J. McNeur, U. Niedermayer, and P. Hommelhoff, Generation and 
Characterization of Attosecond Microbunched Electron Pulse Trains via Dielectric Laser Acceleration, Phys. Rev. Lett. 123, 264803 (2019).

[50] D. S. Black, U. Niedermayer, Y. Miao, Z. Zhao, O. Solgaard, R. L. Byer, and K. J. Leedle, Net Acceleration and Direct Measurement of Attosecond Electron Pulses in a Silicon Dielectric Laser Accelerator, Phys. Rev. Lett. 123, 264802 (2019).

[51] P. B. Corkum and F. Krausz, Attosecond Science, Nat. Phys. 3, 381 (2007).

[52] P. Baum and A. H. Zewail, Attosecond Electron Pulses for $4 D$ Diffraction and Microscopy, Proc. Natl. Acad. Sci. U.S.A. 104, 18409 (2007).

[53] K. E. Priebe, C. Rathje, S. V. Yalunin, T. Hohage, A. Feist, S. Schäfer, and C. Ropers, Attosecond Electron Pulse Trains and Quantum State Reconstruction in Ultrafast Transmission Electron Microscopy, Nat. Photonics 11, 793 (2017).

[54] P. Baum, Quantum Dynamics of Attosecond Electron Pulse Compression, J. Appl. Phys. 122, 223105 (2017).

[55] T. W. Hughes, S. Tan, Z. Zhao, N. V. Sapra, K. J. Leedle, H. Deng, Y. Miao, D. S. Black, O. Solgaard, J. S. Harris, J. Vuckovic, R. L. Byer, S. Fan, R. J. England, Y. J. Lee, and M. Qi, On-Chip Laser-Power Delivery System for Dielectric Laser Accelerators, Phys. Rev. Applied 9, 054017 (2018).

[56] S. Tan, Z. Zhao, K. Urbanek, T. Hughes, Y. J. Lee, S. Fan, J. S. Harris, and R. L. Byer, Silicon Nitride Waveguide as a Power Delivery Component for Dielectric Laser Accelerators, Opt. Lett. 44, 335 (2019).
[57] D. Bar-Lev and J. Scheuer, Plasmonic Metasurface for Efficient Ultrashort Pulse Laser-Driven Particle Acceleration, Phys. Rev. ST Accel. Beams 17, 121302 (2014).

[58] D. Bar-Lev, R. J. England, K. P. Wootton, W. Liu, A. Gover, R. Byer, K. J. Leedle, D. Black, and J. Scheuer, Design of a Plasmonic Metasurface Laser Accelerator with a Tapered Phase Velocity for Subrelativistic Particles, Phys. Rev. Accel. Beams 22, 021303 (2019).

[59] Y. Adiv, H. Hu, S. Tsesses, R. Dahan, K. Wang, Y. Kurman, A. Gorlach, H. Chen, X. Lin, G. Bartal, and I. Kaminer, in Proceedings of the Conference on Lasers and Electro-Optics, Optical Society of America (2021), p. FM1L.6, https://www .osapublishing.org/abstract.cfm?uri=CLEO_QELS-2021FM1L.6.

[60] O. Kfir, Entanglements of Electrons and Cavity Photons in the Strong-Coupling Regime, Phys. Rev. Lett. 123, 103602 (2019).

[61] V. Di Giulio, M. Kociak, and F. J. G. de Abajo, Probing Quantum Optical Excitations with Fast Electrons, Optica 6, 1524 (2019).

[62] V. Di Giulio and F. J. G. de Abajo, Free-Electron Shaping Using Quantum Light, Optica 7, 1820 (2020).

[63] A. Gorlach, A. Karnieli, R. Dahan, E. Cohen, A. Pe'er, and I. Kaminer, Ultrafast Non-Destructive Measurement of the Quantum State of Light Using Free Electrons, arXiv:2012.12069.

[64] R. Dahan, A. Gorlach, U. Haeusler, A. Karnieli, O. Eyal, P. Yousefi, M. Segev, A. Arie, G. Eisenstein, P. Hommelhoff, and I. Kaminer, Imprinting the Quantum Statistics of Photons on Free Electrons, Science 373, eabj7128 (2021). 